\title{
PROGRESS IN THREE-DIMENSIONAL ELASTIC-PLASTIC STRESS ANALYSIS FOR FRACTURE MECHANICS *
}

\author{
N. LEVY, P.V. MARCAL and J.R. RICE \\ Division of Engineering, Brown University, \\ Providence, Rhode Island, 02912, USA
}

Received 14 April 1971

\begin{abstract}
This paper summarizes progress in the development of finite element methods for three-dimensional elasticplastic stress analysis in fracture mechanics. The work is directed toward the study of stress states near flaws such as a part-through crack in a pressure vessel wall, and emphasis is placed on the large scale plastic yielding range The development of a computer program with large computing capabilities is described. Special near crack tip elements and general isoparametric elements are employed in problems with two crack configurations. Preliminary results are given for the elastic analysis of a through crack and for the elastic-plastic analysis of a part-through semi-elliptical crack in a plate.
\end{abstract}

\section{Introduction}

This paper summarizes progress in the development of finite element methods for three-dimensional elastic-plastic stress analysis in fracture mechanics, as a part of the Heavy Section Steel Technology Program. The problems considered deal with crack-like flaws. The part through the thickness surface crack in a pressure vessel wall is typical of those toward which the work is aimed. Further, the materials are regarded as tough, in comparison to those for which linear fracture mechanics is applicable, and hence the emphasis is on the large scale yielding range.

Paris and Sih [1] have reviewed the elastic analysis of cracks, and Irwin [2] has studied the problem of the embedded elliptical crack in an unbounded elastic body as a basis for an approximate treatment of the semi-elliptical surface crack. More recently, Smith and Alavi [3] and Rice and Levy [4] have presented elastic treatments of the part through surface crack in a plate. The former is based on solutions of the three-dimensional elastic field equations with approximate meeting of boundary conditions, whereas the

\footnotetext{
* Research performed under UCCND Subcontract No. 3153 for the Oak Ridge National Laboratory, operated by the Union Carbide Corporation, Nuclear Division, for the U.S. Atomic Energy Commission.
}

latter employs compliance matching to the edge cracked strip in plane strain so as to reduce the part through crack problem to one in two-dimensional plate (or shell) theory.

Elastic-plastic fields near cracks are not yet well understood. Rice [5] has summarized progress in analytical studies, which are limited for the most part to simple models and sometimes also to small scale yielding and to total (as opposed to the proper incremental) stress-strain relations. It is only recently that crack tip singularities have been understood in the two-dimensional plane strain and plane stress models for elastic-plastic materials [5-9]. At another extreme, plastic limit flow fields have been found for plane straining of non-hardening materials in several notched configurations, and McClintock [10] has recently discussed these results. Current work on elastic-plastic fracture includes both fundamental studies on the relation of microstructural separation mechanisms to crack tip toughness [11-13], and also general stress analysis studies aimed at aiding the interpretation and extrapolation of fracture test results in the large scale yielding range.

The finite element method has been widely adopted for this latter task, and results of two-dimensional elastic-plastic analyses have been reported by Marcal and King [14] and Swedlow et al. [15,16], among others. Fracture initiation from the tip of a pre-crack 
is, however, a highly localized phenomenon, and it is not clear that simple finite elements (such as constant strain triangles) can accurately represent the near tip deformation field. This problem of attaining near tip accuracy has been addressed recently by Levy et al. [17] for plane strain and by Hilton and Hutchinson [18] for plane stress, both approaches being guided by the analytical studies of crack tip singularities noted above. In particular, Levy et al. employed singular finite elements adjacent to the crack, capable of reproducing the strain singularity and discrete crack tip opening of the non-hardening material model which they employed, but otherwise adopted the Marcal and King formulation and method of solution. Very little previous work has been done on three-dimensional finite element analysis of cracks with or without refinements for near tip accuracy, although Ayres [19] has presented a finite difference procedure for these problems.

The overall aims of the three-dimensional work under discussion are outlined in the next section. Meaningful problems require enormous numbers of degrees of freedom (10000 is estimated for the part through semi-elliptical crack in a plate), and this requires modifications of the general purpose program developed by Marcal and co-workers $[14,20]$ which are discussed in the following section. Finally, some numerical results are presented. These are preliminary in nature, most of the work to date having been in the areas of program development, element design, and mesh generation.

\section{Aims of three-dimensional finite element studies}

The immediate goal is to develop an elastic-plastic analysis for a plate under tensile loading containing a semi-elliptic surface crack (see fig. 1). The work toward this goal has proceeded in two principal directions:

The first of these is in developing general finite elements and computing procedures for large threedimensional problems. Clearly, in a configuration such as that of fig. 1 a large number of elements is necessary, especially in view of the strong gradients anticipated along the crack front. This, coupled with the fact that the master stiffness matrix has a large bandwidth (typically 500 degress of freedom for

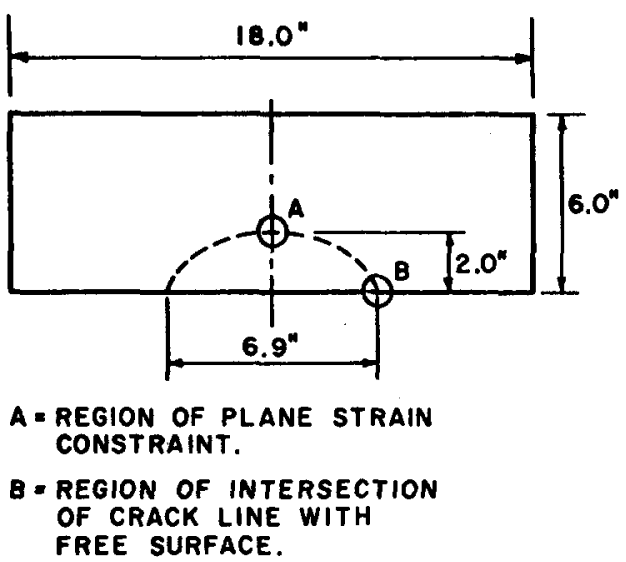

Fig. 1. Dimensions of the cross-section and of the partthrough semi-elliptical crack of a flawed tension specimen.

cubic elements) requires many steps in the optimization of the computing procedures which are discussed in the next section. The general finite element which has been employed is the three-dimensional isoparametric element [21]. This is illustrated in fig. 2, and is obtained by arbitrarily positioning in $(x, y, z$, (or physical) space the points corresponding to the corners of a unit cube in $(\xi, \eta, \zeta)$ space. The mapping is given by equations of the form

$$
x=x_{A}(1-\xi)(1-\eta) \zeta+x_{B} \xi(1-\eta) \zeta+\ldots,
$$

where $x_{\mathrm{A}}, x_{\mathrm{B}}, \ldots$ are $x$ coordinates of the nodes $A, B, \ldots$. The rectangular parallelepiped element is the

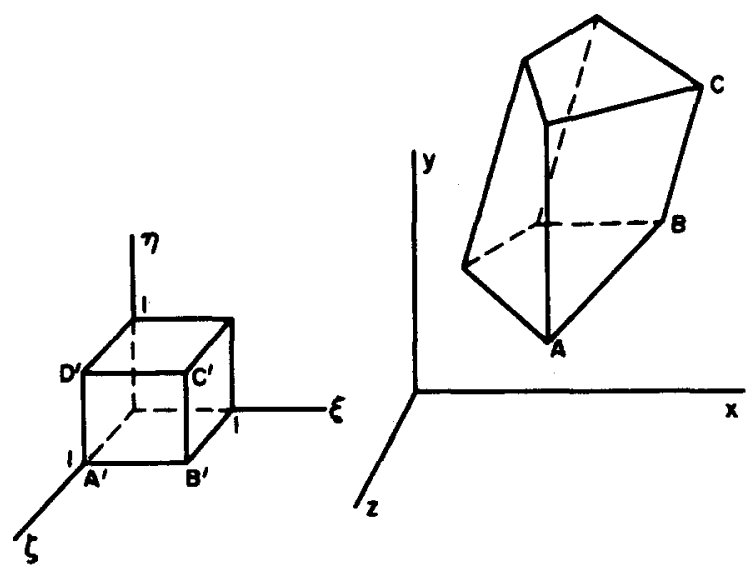

Fig. 2. The distorted cubic element in the physical space $x, y, z$ and its map in the $(\xi, \eta, \xi)$ space. 
simplest special case and is used extensively, for example, at distances far from the crack surface disturbance in fig. 1 . The element is versatile by virtue of its possible distortions, as in fig. 2. Assumption of a similar form for displacements, e.g.

$$
u=u_{\mathrm{A}}(1-\xi)(1-\eta) \zeta+u_{\mathrm{B}} \xi(1-\eta) \zeta+\ldots,
$$

where $u_{\mathrm{A}}, u_{\mathrm{B}}, \ldots$ are nodal point displacements, assures continuity across element boundaries. This element has been employed in preliminary studies of the semi-eliptical surface crack specimen, to be discussed subsequently.

As has been noted earlier, routine finite element solutions will not necessarily give sufficient accuracy very near the crack tip where continued fracturing is imminent. Thus, a second direction in the threedimensional studies has been on developing special finite elements and procedures for accurate reproduction of near tip singular behavior. It was necessary to employ a simpler configuration than the surface crack for this purpose, and therefore the through crack in a plate of finite thickness was examined (fig. 3). This problem is also of substantial interest in itself, and it contains, in simpler form, many of the features present for the semi-ellipse. For example, point $A$ on the semi-ellipse in fig. 1 and the middle region of the through crack in fig. 3 both will be under conditions approximating plane strain constraint, whereas point B on the semi-ellipse and the similar point on the through crack both lie on surfaces for which the normal and shear stresses are zero. The near tip analysis for this case is being pursued under small scale yield.

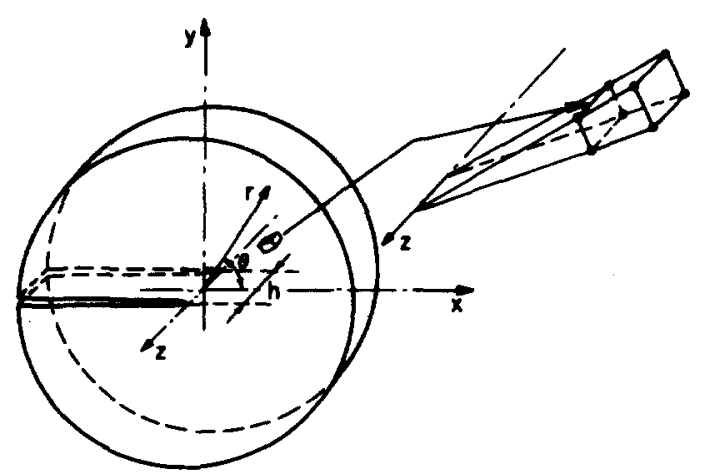

Fig. 3. Cylindrical cut-out used to analyse the three-dimensional through crack in a plate, with a typical finite element; $h$ is the thickness of the plate, and $x-y$ is the mid-plane. ing boundary conditions $[5,6,17]$. That is, a set of surface stresses given by the characteristic twodimensional elastic singularity is applied to the outer radial surface. The elements employed are bounded by coordinate surfaces of a cylindrical polar system as in fig. 3. The corresponding two-dimensional version is, in fact, the same element which Levy et al. [17] found suitable for an accurate near tip treatment of the plane strain problem for a non-hardening elasticplastic material.

The ultimate plans call for combining special near tip elements, designed for accurate reproduction of singularities and large stress gradients, with the general purpose three-dimensional elements of the isoparametric type. This step has not yet been taken, and we report here preliminary results only for the two separate directions of study being taken at present. It should be remarked that one possibility is that the isoparametric elements may be arrayed in polar fashion about the crack tip. This is accomplished by mapping two parallel edges (say $A^{\prime} B^{\prime}$ and $C^{\prime} D^{\prime}$ in fig. 2) into a single line in $(x, y, z)$ space, giving an element with properties similar to those of a cylindrical polar element having a zero inner radius.

\section{Description of the computer program}

The computer program follows the general scheme outlined by Marcal and King [14]. The major effort was directed at developing a computer program with sufficient capacity to handle the large number of degrees of freedom associated with the three-dimensional crack problems described earlier.

To describe the program, we define the pertinent quantities derived in detail in [20] and write down the expression for the incremental stiffness matrix $[k]$ of an element,

$$
[k]=\int_{V}[\alpha]^{\mathrm{T}}[B]^{\mathrm{T}}[D][B][\alpha] \mathrm{d} V,
$$

where the integration is carried out over the volume $V$ of an element. The $[\alpha]$ matrix is the generalized displacement transformation matrix which transforms increments of nodal displacements $\mathrm{d}[u]$ to increments of the undetermined coefficients $\mathrm{d}[a]$ which describe the displacement function. $[B]$ is the differential operator which transforms the increments of 
undetermined coefficients $\mathrm{d}[a]$ to increments of strain $\mathrm{d}[e]$ and $[D]$ is the incremental stress-strain relation for a Prandtl-Reuss material obeying the Mises yield criterion.

The general finite elements which have been used are the three-dimensional isoparametric element and the three-dimensional polar elements described above. In both cases, the stiffness matrix is evaluated by a trapezoidal integration procedure using eight representative points. Numerical integration is needed because the differential operator $[B]$ is a function of position.

In order to cope with the size of the problem it was necessary to use auxiliary storage to store the master stiffness matrix (with packed non-zero coefficients) and the element data, such as stresses and strains. The element data is used in a sequential manner and so it can be retrieved economically. The displacement and nodal point coordinate vectors, however, are used in a random manner and it was decided to keep these quantities in core. This has resulted in an upper limit of about 10000 degrees of freedom that can be solved on an IBM 360 computer with about 125 kwords of core.

Much effort has been devoted to optimizing the input output (I.O.) operations. A certain amount of "fine tuning" was necessary in order to balance the cost due to either the generation of a quantity or its retrieval from the backing store. This "fine tuning" is particularly important for the large parallel operation machines, such as the IBM 360/90 and CDC 6600. In order to compute efficiently on these machines, options were provided to avoid computing the quantities which could be regenerated more economically. It was found that, even for these machines, it was still advantageous to store the elastic stiffness of each element rather than to regenerate it.

The computer program is best described by dividing it into three computing stages. The first is that of data input storage. Here parameters defining the type of elements to be used and the nodal point connectivity are read in and distributed to the appropriate storage unit. The program then performs an elastic solution. The $[a],[B],[D]$ and $[k]$ matrices are formed. An element repeat feature is included to avoid repetitive generation of element matrices. The stiffness matrices are then assembled into a master stiffness matrix. Because of the generally large band- width of the master stiffness matrix (typically 500 degrees of freedom), the solution is carried out by a Gauss-Seidel iteration scheme, with a pre-determined over-relaxation factor (usually between 1.8 to 1.85). An option allows for the calculation of an optimized over-relaxation factor [22]. The stresses and strains are then calculated and the results are scaled elastically to cause the most highly stressed element to yield. This completes the elastic calculations and an incremental elastic-plastic analysis. is performed next. An incremental load of about 0.1 the first yield load is applied. Each element is examined in turn to see if yielding will occur; where yielding occurs a new stiffness matrix is generated. The old stiffness matrix for the element is retrieved from backing store and it is then used for updating the master stiffness matrix. The test for yielding of an element is made with the aid of an estimate of the strain increment. For proportional loading the strain estimate is based on the results of the last load increment. The master stiffness matrix is then used to solve for the increment of displacement. An option is provided to recalculate the master stiffness matrix with an improved estimate of the strain increment. When convergence of the displacement increments is obtained, the calculations of strains and stresses are performed and the computation is returned for another elastic-plastic increment of load. It is of interest to note here that, for the size of problem solved, it was found necessary to have restart capabilities at selected points within the program. The data for restart are stored successively for the various increments so that the user can prescribe a restart at any increment of the solution and thus minimize any recalculation of data.

To conclude this brief description of the computer program we note the blocking of our over-relaxation procedure to reduce the 1.0 . calls. The program reads $n$ rows of the master stiffness matrix and reiterates for a prescribed number of times $m$ while the next "block" is being prepared for transfer into core (using the FIND statement). For the IBM machines, because of limitations on data transfer, $n$ and $m$ were taken equal to 60 and 20 . Further I.O. savings were effected by implementing the various suggestions outlined in [23]. The I.O. times are usually of an order of magnitude larger than that of the arithmetical compute times (C.P.U.) so that the importance of attention to. I.O. details cannot be overemphasized. The experi- 
ence gained in using the program is passed on to other users by encasing it in a program to punch out job control cards for users which optimize the specification of the various data sets, record length, block size, number of buffers, etc.

\section{Mesh generation procedures}

Automatic mesh generation is mandatory in threedimensional problems. Programs have been written to generate the finite element description of a through crack using the polar elements and a tensile plate with a semi-elliptic crack using a combination of cubic and isoparametric elements. Only three input cards are required to generate all the data and loads for the problem to be solved.

\section{Three-dimensional through crack problem}

The results of an initial elastic analysis for the through crack in a plate are discussed here. The analysis was done in the interest of studying near tip element design as noted earlier, but the results may also be of interest for the light they shed on the actual three-dimensional nature of what is frequently treated as a two-dimensional problem. In particular, if $(r, \theta)$ are polar coordinates in the $(x, y)$ plane of fig. 3 , then two-dimensional (plane stress) elastic solutions result in near tip singular stress states of the form

$$
\left\{\begin{array}{l}
\sigma_{r r} \\
\sigma_{r \theta} \\
\sigma_{\theta \theta}
\end{array}\right\}=\frac{K_{I} \cos \left(\frac{1}{2} \theta\right)}{(2 \pi r)^{1 / 2}}\left\{\begin{array}{l}
1+\sin ^{2}\left(\frac{1}{2} \theta\right) \\
\sin \left(\frac{1}{2} \theta\right) \cos \left(\frac{1}{2} \theta\right) \\
1-\sin ^{2}\left(\frac{1}{2} \theta\right)
\end{array}\right\}
$$

where $K_{I}$ is the opening mode stress intensity factor and where $\theta$ is measured from zero on the $x$-axis ahead of the crack tip. For a first treatment of actual three-dimensional effects, we have analyzed a cut-out circular segment as in fig. 3 , having a radius which is six times the plate thickness. The two-dimensional solution may be expected to be valid at this distance from the tip, and therefore, as boundary conditions we apply stresses $\sigma_{r r}$ and $\sigma_{r \theta}$ from the above equation to the outer radius of the cut-out.

The cylindrical polar elements are shown in fig. 3 .
These were stacked in six layers of equal height through the half thickness. The angular range of each element was $15^{\circ}$, so that there were 12 wedges of elements in the upper half of the cut-out, and there were 15 annular rings of elements, including the ring immediately adjacent to the crack tip which had a zero inner radius and an outer radius of $R_{\mathrm{o}}=0.05 \mathrm{~h}$ (where $h$ is the total plate thickness). Thus, in all, there were 1080 elements in the quarter segment required (by symmetry) for analysis, resulting in 1456 nodal points and 4048 degrees of freedom ( 3 per nodal point, minus the number of nodal displacement components set to zero on symmetry planes). For additional details, see $[20,24]$.

While only the elastic analysis is under discussion here, it should be remarked that the governing feature in selection of the elements was their ability to simulate anticipated near tip behavior in the elastic-plastic range. Indeed, analytical studies [6-9] on twodimensional crack problems have shown the following features of the near tip field in elastic-plastic materials which are idealized as non-hardening. The cartesian displacement components at the crack tip vary with orientation angle $\theta$. That is, different sets of displacements may result for different radial lines along which the crack tip is approached, and there is a finite opening displacement at the crack tip. Also, in any angular range in which the displacements do vary with $\theta$, the plastic strains exhibit a $1 / r$ singularity at the crack tip. These are features of solutions to the conventional equations of elastic-plastic stress analysis, in which finite changes in geometry are neglected. Rice and Johnson [13] studied finite geometry changes at the crack tip in plane strain and found that the region of large strain or rotation was highly localized, having linear dimensions of the order $5\left(\sigma_{0} / E\right) r_{\mathrm{p}, \max }$ for contained plastic yielding, where $\sigma_{0}$ is the yield stress, $E$ is Young's modulus, and $r_{\mathrm{p}, \mathrm{max}}$ is the maximum extent of the plastic zone. They also showed that the crack tip singularity field, from the conventional solution neglecting finite geometry changes, could be employed to set boundary conditions on a local analysis of large plastic blunting at the tip.

This suggests that some care is necessary in the design of near tip finite elements if these important near crack tip features are to be revealed and characterized quantitatively in the numerical solution. Thus, 
Levy et al. [17] employed two-dimensional polar elements for which the three-dimensional generalization is shown in fig. 3, those members of the polar set immediately adjacent to the crack were still regarded as having four independent nodal points, even though two of the nodes initially occupied coincidental spatial positions at the crack tip. This resulted in making possible displacement fields having near tip behavior of the proper form, and the displacement assumption within the element necessarily led to the $1 / r$ strain singularity whenever different displacements were assigned to the coincidental nodes. The three-dimensional elements of fig. 3 have similar features, but the costly elastic-plastic analysis has not yet been carried to completion, pending further preliminary studies on mesh refinement near the intersection of the crack with the free surface.

Fig. 4 shows the variation of stresses with distance from the crack tip according to mid-element stresses in the wedge of elements between $0^{\circ}$ and $15^{\circ}$ in the layer immediately adjacent to the middle surface of

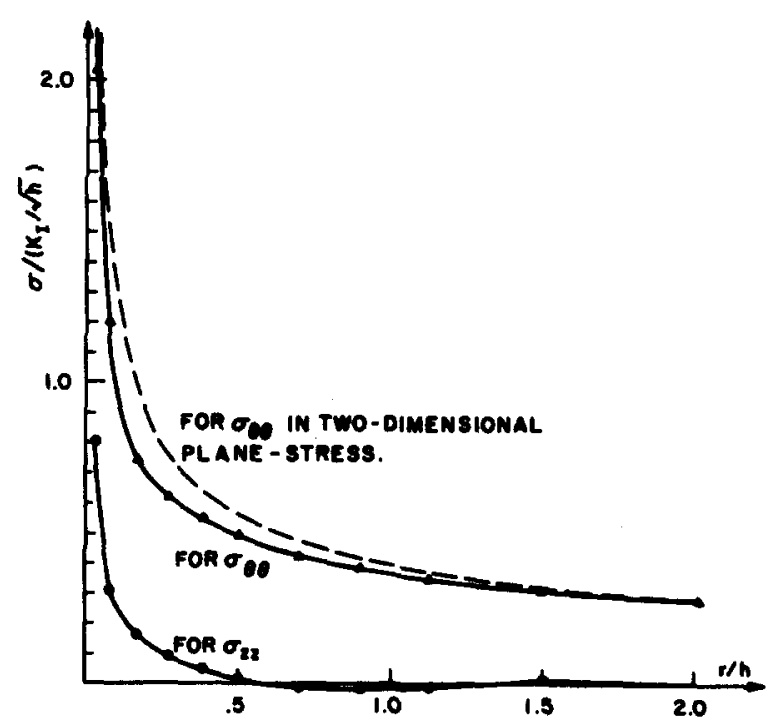

Fig. 4. The midelement values of $\sigma_{z z}$ and $\sigma_{\theta \theta}$, calculated elastically as functions of the distance from the crack in the elements ahead of the crack, immediately adjacent to the mid-plane. Solid lines represent results of the numerical calculations, wheras the dashed line is $\sigma_{\theta \theta}$ from the two-dimensional elastic plane-stress solution. The stresses are made dimensionless through division by $\kappa_{I} / \sqrt{h}$, and the distance $r$ from the crack is made dimensionless through division by $h$. $\kappa_{I}$ is the stress intensity factor in plane stress, $h$ is the total thickness of the plate. the plate. A Poisson ratio $\nu=0.3$ is taken in the calculations. The scaling of stress distance in the figure represents a proper non-dimensional form for the results, at least when the radius of the cut-out is large compared to its thickness. The results for $\sigma_{\theta \theta}$ may be compared with predictions of the above two-dimensional plane stress solution as shown by the dashed line. Both coincide beyond a distance of $2 h$ from the crack tip. However, as we approach the tip, the three-dimensional numerical values are somewhat lower than those predicted by the two-dimensional solution. An examination of the slopes suggests, on the other hand, that $\sigma_{\theta \theta}$ could attain a higher value than the two-dimensional prediction at very small distances from the tip. The stress component $\sigma_{z z}$ (in the thickness direction) is also shown. This is, of course, taken as zero in the two-dimensional plane stress treatment. It is seen that this stress falls off very rapidly, being negligible beyond a distance of $0.5 h$ from the crack tip. Thus, the region of significant departure from plane stress conditions is highly localized.

Fig. 5 shows mid-element stresses as a function of distance from the middle surface of the plate, for the column of elements immediately adjacent to the crack tip within the $0^{\circ}$ to $15^{\circ}$ wedge. The midpoints

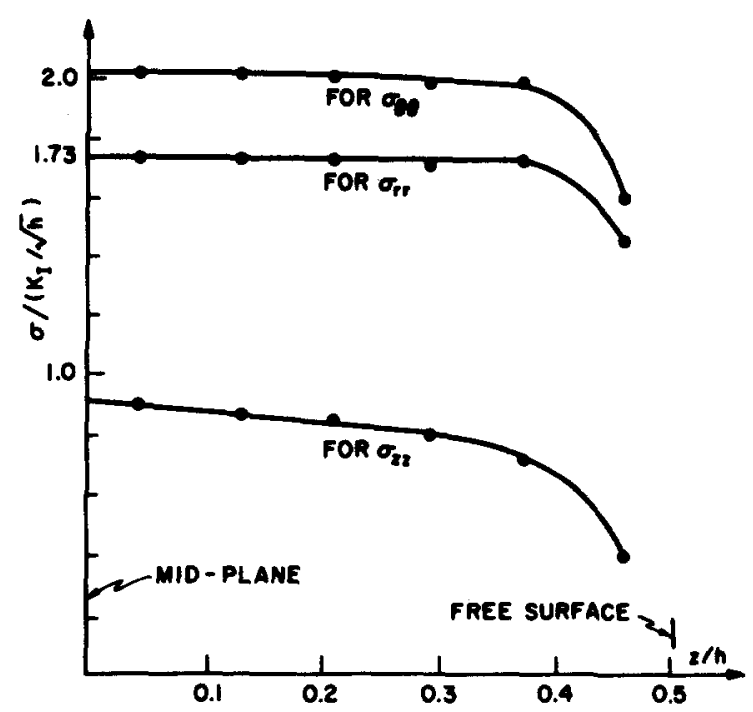

Fig. 5. The mid-element values of $\sigma_{\theta \theta}, \sigma_{r,}, \sigma_{z z}$, calculated elastically as functions of the distance $Z$ from the mid-plane, in the elements nearest to and ahead of the crack. Stresses and the distance are made non-dimensional as in fig. 4 . 
of these elements are at a radius of $0.025 h$ from the tip. It is seen that the in-plane stresses $\sigma_{r r}$ and $\sigma_{\theta \theta}$ are very nearly constant through the thickness, except for a rapid variation near the free surface. Also, $\sigma_{z z}$ varies only slightly before taking a steep plunge to the values of zero required at the surface. The shear $\sigma_{r z}$, which must vanish at the middle surface and at the free surface, is found to be negligible throughout the thickness.

The equation for the strain in the thickness direction is

$$
\epsilon_{z z}=\frac{1}{E}\left[\sigma_{z z}-\nu\left(\sigma_{r r}+\sigma_{\theta \theta}\right)\right]
$$

and one expects that $\epsilon_{z z}$ will be bounded along the crack tip (except possibly at its intersection with the plate surface). On the other hand, the in-plane stresses are unbounded at the tip, and hence at least the in-plane strains are singular. This observation suggests that the elastic singularity at a crack tip in a three-dimensional problem must always be of the form of the two-dimensional plane strain singularity as noted by Irwin [25]. The ratio

$$
\frac{\sigma_{z z}}{\nu\left(\sigma_{r r}+\sigma_{\theta \theta}\right)}
$$

may be defined as the degree of plane strain, it being unity when $\epsilon_{z z}$ is either zero or bounded at the site of a stress singularity, and zero under plane stress conditions when $\sigma_{z z}=0$. One expects a degree of plane strain of unity along the crack tip, but this must decrease very rapidly to zero at the surface of the plate, suggesting a fan-like corner singularity at the intersection of the crack and the free surface. Presumably, the rapid variations near $z=1 / 2 h$ in fig. 5 result from passing through this fan on a straight line in the $z$-direction. The results of fig. 4 suggest a degree of plane strain of essentially zero at distances from the tip greater than a half thickness, even in the middle plane of the plate. From the data of fig. 5 , the degree of plane strain averages about 0.8 for the three element closest to the middle surface. However, examination of mid-element stresses for those at greater distances from the tip shows that the numerical results extrapolate approximately to unity at the tip.

\section{Elastic-plastic analysis of tensile specimen with semi-elliptic crack}

As a first step towards the understanding of the part-through surface crack, a tensile specimen with a semi-elliptic crack was chosen for experimental study under the HSST project. The crack line configuration and the cross section of the specimen are shown in fig. 1 . The same specimen is treated here by the finite element method, the objective being to better interpret experimental results in view of the numerical solution. In this section we report on a preliminary analysis of such a specimen.

Because of symmetry, only one quadrant of the actual specimen was considered. The quadrant is divided into 1152 elements distributed evenly in 9 layers varying in thickness from $0.25^{\prime \prime}$ adjacent to the crack plane to $5.0^{\prime \prime}$ adjacent to the plane of loading. Fig. 6 shows the distribution of the nodal points and elements in the plane of the crack, which is identical for all 9 slices. For the most part, the nodal points are defined by the intersection of rays emanating from the center of the coordinates system with curves defined by

$$
(x / a)^{p}+(y / b)^{2}=1 .
$$

Note that with $p=2, a=3.45^{\prime \prime}$ and $b=2.0^{\prime \prime}$, the curve matches exactly the semi-elliptic crack contour. On the other hand, when $a=9.0^{\prime \prime}$ and $b=6.0^{\prime \prime}$, the curve can match the outer boundary of the specimen as close as we wish by increasing the value of $p$.

In all 1152 elements and 1500 nodal points are used, resulting in 4111 degrees of freedom ( 3 per nodal point minus the number of nodal displacement components set to zero on symmetry planes).

An elastic-perfectly-plastic Misses material was assumed with a Poisson's ratio of 0.3 in the elastic range. The specimen is loaded in tension by imposing a uniformly distributed tensile stress on a plane parallel to the plane of the crack at a distance equal to 2.5 times the width of the specimen.

\section{Elastic solution}

The elastic solution is first obtained and scaled so that the element with the highest equivalent stress (as 
based on the mid-element values) is at yield. This corresponds to an applied stress of $0.617 \sigma_{0}$, where $\sigma_{0}$ is the tensile yield, for the element size employed. Six load increments, each equal to 0.1 of the elastic load, are added to study the elastic-plastic range. At the sixth increment, the plastic limit load is reached in the sense that no solution can be found for an additional increment, even when the increment size is reduced by a half. The resulting applied stress at limit load is therefore approximately $0.987 \sigma_{0}$, corresponding to an average stress of approximately $1.09 \sigma_{0}$ on the net section of the plane containing the crack. It is not expected that the computed value for the limit will be sensitive to the average size of the elements used. The value obtained numerically is therefore a reasonably accurate estimate of the actual ideally plastic limit load in spite of the coarse mesh used.

The elastic solution indicates that element 51 in fig. 6 is the first one to yield. The element lies in front of the crack line along the minor axis.

Fig. 7 shows the variation with the angle measured from the $x$-axis, of the degree of plane strain at the crack tip defined by the ratio,

$$
\frac{\sigma_{\theta \theta}}{v\left(\sigma_{r r}+\sigma_{z z}\right)}
$$

where $\sigma_{\theta \theta}, \sigma_{r r}$ are the stresses tangential and normal to the crack line respectively, and $\sigma_{z z}$ is the stress perpendicular to the plane of the crack.

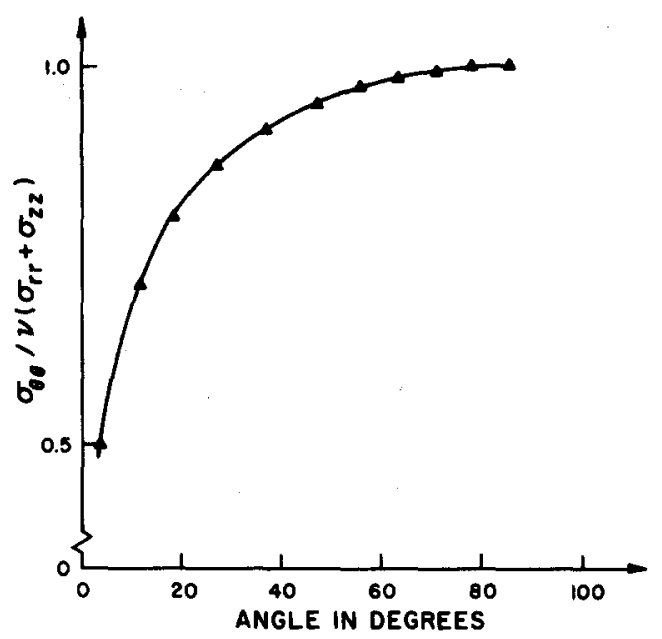

Fig. 7. Variation of the degree of plane strain at the crack line with the angle measured from the $x$-axis (see fig. 6).

Clearly, this ratio attains a value of 1.0 at the crack tip along the minor axis indicating a state of plane strain in this region, dropping rapidly towards the value zero corresponding to a state of plane stress in the region where the crack line meets the free surface.

Figs. 8 and 9 show the variation of $\sigma_{z z} / \sigma_{0}$ with distance from the crack tip, in the elements along the major and minor axes of the semi-elliptic crack respectively at the elastic and the limit loads. The value

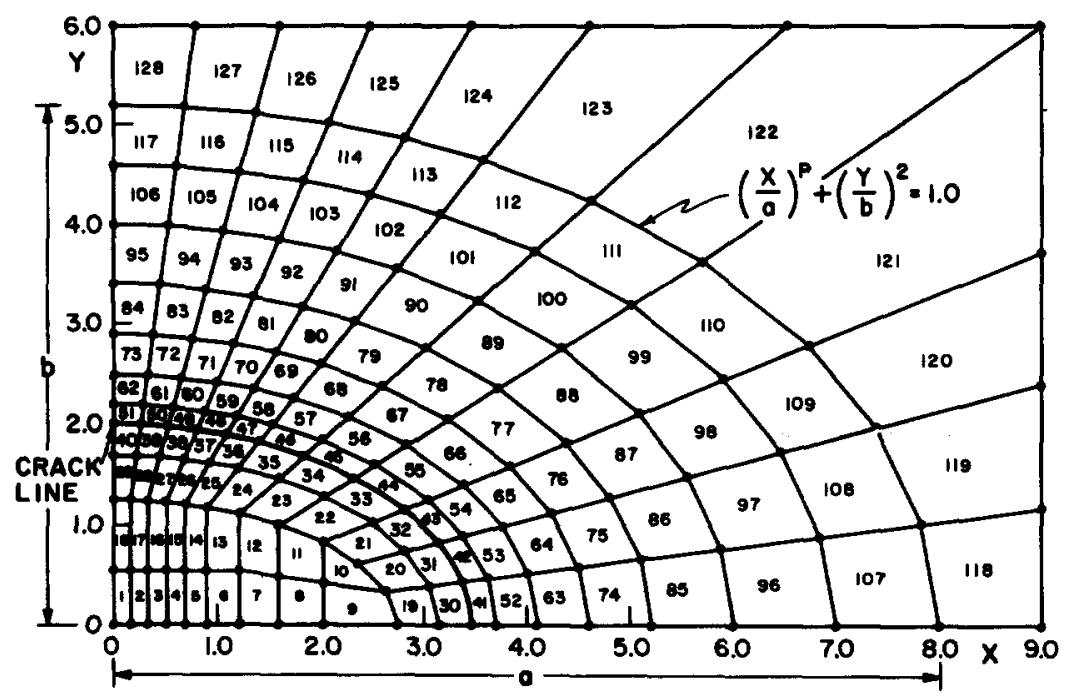

Fig. 6. The elements adjacent to the plane of the crack in the part-through surface flawed tension specimen. 


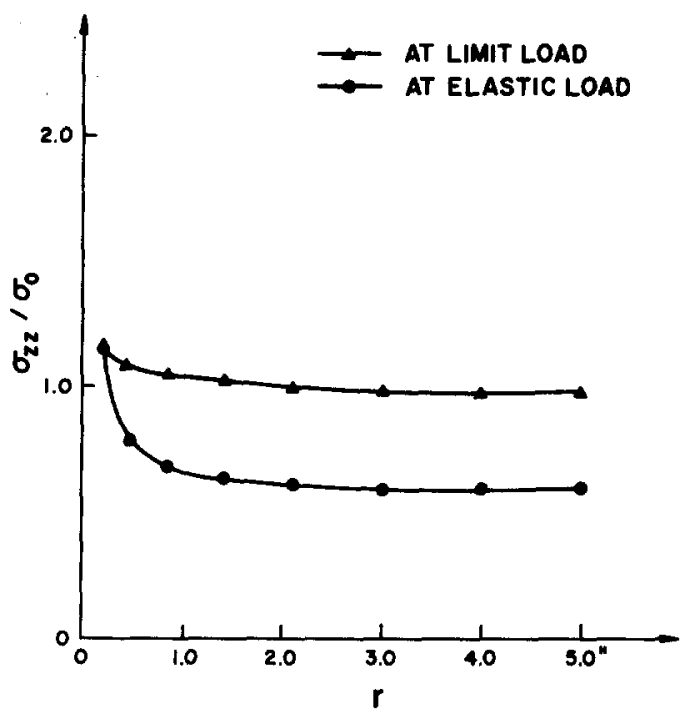

Fig. 8. Variation of the ratio $\sigma_{2 z} / \sigma_{0}$ in the elements along the major axis of the semi-elliptic crack with the distance $r$ from the crack, at the elastic and the limit loads. $\sigma_{2 z}$ is the stress perpendicular to the plane of the crack $\sigma_{0}$ is the yield stress of the material.

of the ratio $\sigma_{z z} / \sigma_{0}=1.15$ in the element nearest to the crack tip along the major axis beyond the elastic load suggests that the mode of yielding in this element is that of an in-plane shear. Along the minor

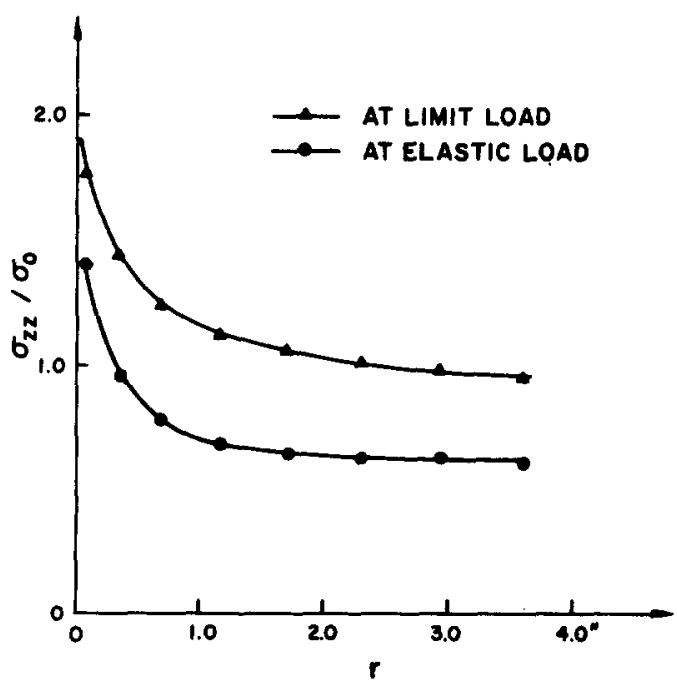

Fig. 9. Same as for fig. 8, for the elements along the minor axis. axis, the value $\sigma_{z z} / \sigma_{0}=1.45$ at the elastic load in the element nearest to the crack tip exhibits the degree of tri-axiality attained in this region.

\section{The stress intensity factor along the minor axis of the crack}

Estimation of the stress intensity factor at the mid-point of a part-through crack is of considerable practical importance, since it is in the central section that the breaking through to the far plate surface is imminent. To calculate its value from the numerical results, we use three methods.

1. In the first method, we use the expression from the singular analytic solution for the near crack tip stress state and write

$$
\sigma_{z z} \sqrt{2 \pi r} \rightarrow K \quad \text { as } \quad r \rightarrow 0
$$

where $K$ is the stress intensity factor and $r$ is the distance from the crack tip

The value of $\sigma_{z z} \sqrt{2 \pi \mathrm{r}}$ may be calculated from the numerical results as a function of $r$ and extrapolated to $r=0$ to give an estimate of $K$ at the crack tip. Fig. 10 shows the variation of $\sigma_{z z} \sqrt{2 \pi r} / \sigma_{\infty}$ with $r ; \sigma_{\infty}$ being the imposed average tensile stress. The extrapolated value at $r=0$ is found to be equal to 1.64 $1.80 \mathrm{in}^{1 / 2}$.

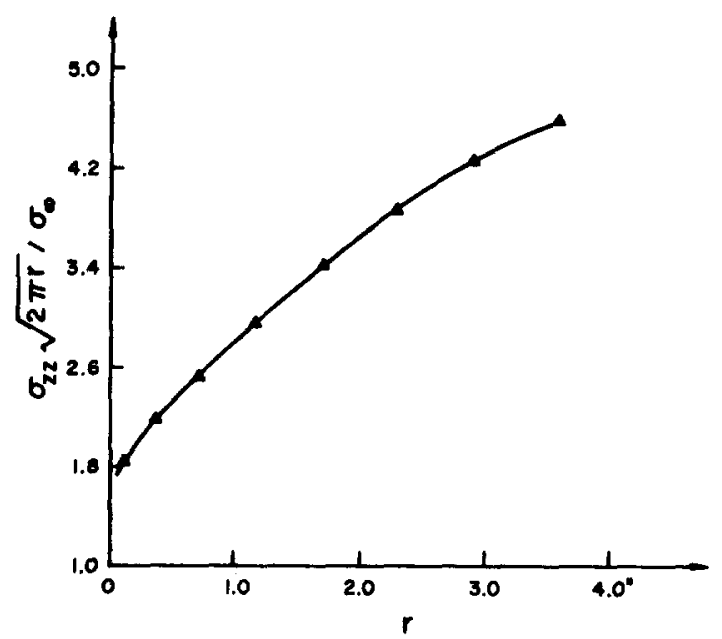

Fig. 10. Variation of the ratio $K / \sigma_{\infty}=\sigma_{z z} \sqrt{2} \pi r / \sigma_{\infty}$ with the distance $r$ from the crack in the elements along the minor axis. $K$ is the stress intensity factor. $\sigma_{\infty}$ the loading stress. $\sigma_{z z}$ the stress perpendicular to the plane of the crack. 
2. The second method consists in computing the tensile stress averaged over the thickness of the plate $-\sigma_{\mathrm{a}}$, for the elements along the minor axis, and the averaged moment of the forces in these elements with respect to an axis parallel to the $x$-axis (see fig. 6) and passing through the middle of the specimen thickness $-m_{\mathrm{a}}$ (when converted to a nominal bending stress). The stress intensity factor is then computed from the following expression suggested by Rice and Levy [4] :

$$
K=h^{1 / 2}\left[\sigma_{\mathrm{a}} g_{\mathrm{t}}+m_{\mathrm{a}} g_{\mathrm{b}}\right],
$$

where $g_{t}(\xi)$ and $g_{b}(\xi)$ are dimensionless functions of the ratio $\xi$ of the crack depth to the plate thickness. The functions are taken from the boundary collocation solution by Gross and Srawley [26] :

$$
\begin{aligned}
& g_{t}(\xi)=\xi^{1 / 2}\left[1.99-0.41 \xi+18.70 \xi^{2}\right. \\
& \left.-38.40 \xi^{3}+53.85 \xi^{4}\right] \\
& g_{b}(\xi)=\xi^{1 / 2}\left[1.99-2.47 \xi+12.97 \xi^{2}\right. \\
& \left.-23.17 \xi^{3}+24.80 \xi^{4}\right] .
\end{aligned}
$$

From the numerical solution and equations $(10)$ and (11), the value of $K / \sigma_{\infty}$ turns out to be equal to 1.59 in $^{1 / 2}$, which agrees with the results of the first method within approximately $12 \%$.

The reduction of the stress intensity factor due to the moment induced by the uncracked region may be estimated by comparing the computed stress intensity factor $K$, to the stress intensity factor $K_{\infty}$ for a notched plate in plane strain loaded in pure tension and with the same ratio of crack depth to plate thickness i.e. $\xi=1 / 3$. From [4], we have:

$$
K_{\infty} / \sigma_{\infty}=h^{1 / 2} g_{\mathrm{t}}(1 / 3)=4.485 \mathrm{in}^{1 / 2} .
$$

Thus $K / K_{\infty}=0.355$ for method (2), and 0.365 to 0.401 for method (1).

It is clear, therefore, that the influence of the induced moment is quite significant.

3. The third method follows the approximate treatment presented by Rice and Levy in [4], for the tensile stretching and bending of a plate containing a surface crack penetrating part through the thickness. The part through crack is represented as a continuous line spring which has both stretching and bending resistance, its compliance coefficients being chosen to match those of an edge cracked strip in plane strain, and the problem is treated as one in two-dimensional plane stress and plate bending. The mathematical formulation reduces to two coupled integral equations for the thickness averaged force and moment per unit length along the cracked section. These have been solved numerically using the crack configuration of the specimen studied, where the crack depth to plate thickness ratio $\xi=1 / 3$ and the crack length to plate thickness ratio is equal to 1.15 . The value of $K / K_{\infty}$ is found to be equal to 0.386 which is within $4 \%$ and $10 \%$ of the values obtained by methods (1) and (2) respectively. This agreement suggests that the model used in [4] is a good approximation even for not very shallow cracks as the one used in the analyzed specimen.

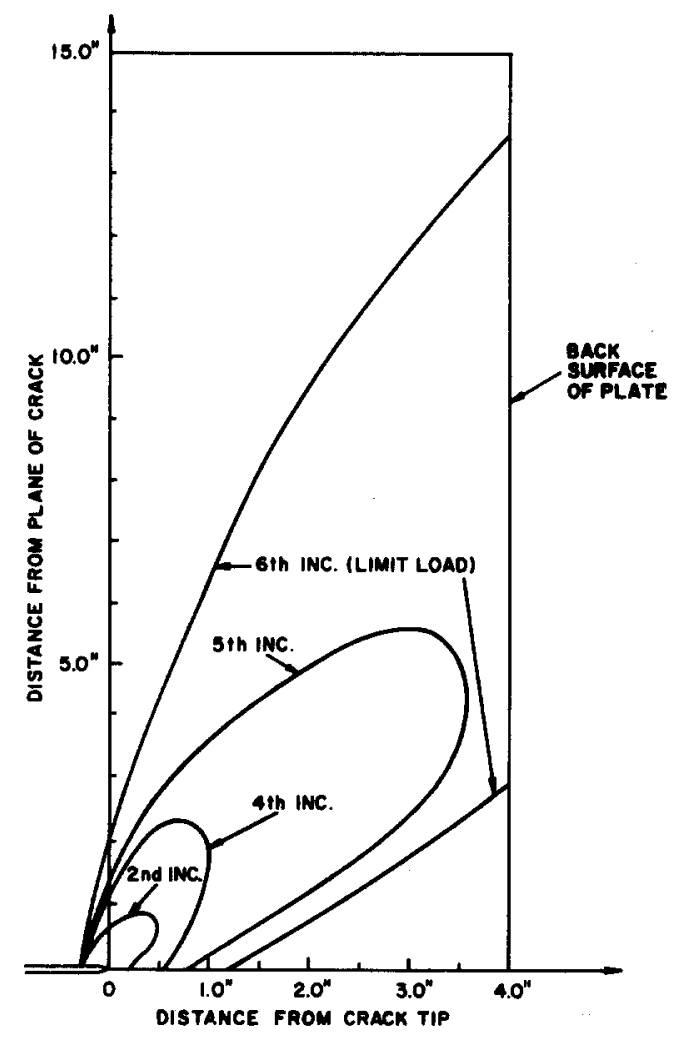

Fig. 11. Plastic zone fronts in the mid-plane at various load increments. 


\section{Plastic solution}

Figs. 11 and 12 show the extents of the plastic zones at the various load increments at the mid-plane and the free surface respectively. It can be seen that at the free surface the plastic zone is tilted towards the plane of the crack, while in the mid-plane an elastic wedge at the far off surface of the plate persists even at the limit load.

In figs. 8 and 9 the variations of $\sigma_{z z} / \sigma_{0}$ with distance from the crack tip are shown for the elements along the major and minor axes respectively at the limit load. It is seen that along the major axis, the yielding mode for the first two elements nearest to the crack tip is that of a through the thickness shear. Farther away from the crack, the elements deform in pure uniaxial tensile mode of yielding. Along the minor axis this ratio tends to the value of 2.0 at the crack tip as compared to 3.0 in the plane strain crack problem as shown by Levy et al. in [17].

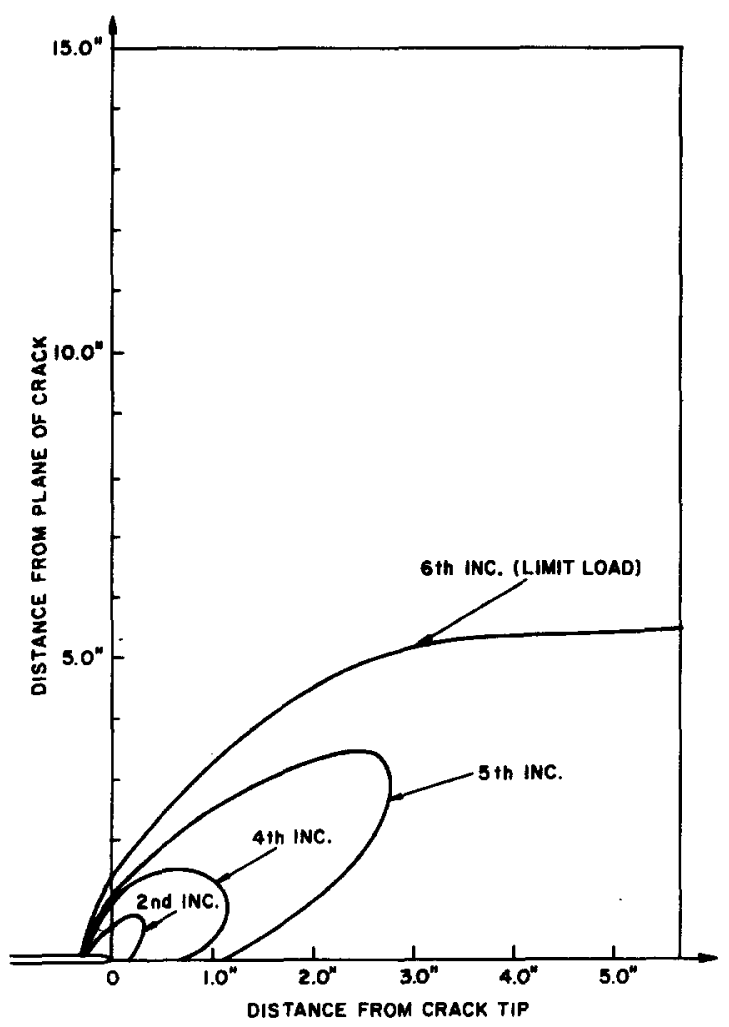

Fig. 12. Plastic zone fronts in the free surface at various load increments.

\section{Crack opening}

The elements used do not allow a discontinuous field of displacements at the crack tip and hence the crack tip opening displacement could not be obtained in the manner of Levy et al. in [17]. However, a measure of crack opening near the tip is obtained from the separation of the crack surface measured at point along the minor axis at a distance of $0.3^{\prime \prime}$ from the crack tip. Fig. 13 shows the variation of the crack surface separation properly non-dimensionalized, as a function of the ratio $\left(K / K_{0}\right)^{2}$ where $K$ is the current stress intensity factor and $K_{0}$ is the stress intensity factor at first yielding. It is clear that a linear relationship between the two variables is obtained up to $95 \%$ of the limit load and, while the straight line does not extrapolate to zero at zero load, it is of interest that a similar linear relation results for small scale yielding.

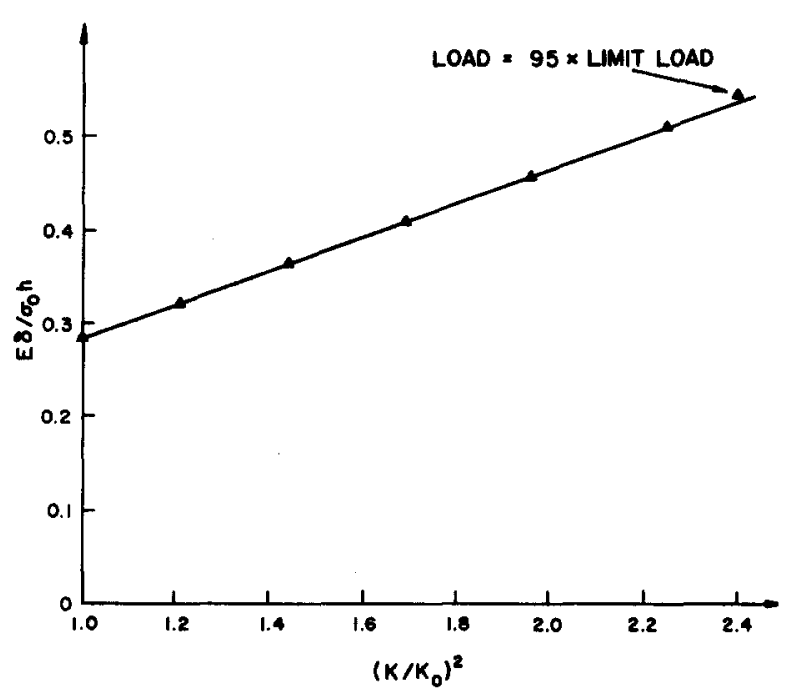

Fig. 13. Variation of the crack surface opening with the load. $\delta$ is the crack opening. $E$ the Young's modulus. $\sigma_{0}$ the yield stress. $h$ the thickness of the plate. $K$ the current stress intensity factor. $K_{0}$ the stress intensity factor corresponding to the elastic load.

\section{Acknowledgment}

The work was carried out under the USAECsponsored Heavy Section Steel Technology Program at the Oak Ridge National Laboratory (Union Car- 
bide Corporation, Nuclear Division subcontract 3153). The authors are indebted to J.G. Merkle and F.J. Witt of the Oak Ridge National Laboratory for useful discussions. The assistance of Miss C.D. Paulk, of the same Laboratory, with the computing aspects of the project is also gratefully acknowledged.

\section{References}

[1] P.C. Paris and G.C. Sih, Stress Analysis of Cracks, in: Fracture Toughness Testing and Its Applications, ASTM, Philadelphia, STP-381, p. 30, 1965.

[2] G.R. Irwin, Crack-Extension Force for a Part-Through Crack in a Plate, ASME J. Appl. Mechanics, Vol. 29, p. $651,1962$.

[3] F.W. Smith and M.J. Alavi, Stress-Intensity Factors for a Part-Circular Surface Flaw, ASME, Proceedings of the First Internation Conference on Pressure Vessel Technology, 1969.

[4] J.R. Rice and N. Levy, The Part-Through Surface Crack in an Elastic Plate, ASME J. Appl. Mech. - in press.

[5] J.R. Rice, Mathematical Analysis in the Mechanics of Fracture, Chapter 3 of Fracture, An Advanced Treatise, Volume II (H. Liebowitz, ed.) Academic Press, New York, 1968.

[6] J.R. Rice, A Path Independent Integral and the Approximate Analysis of Strain Concentration by Notches and Cracks, J. Appl. Mech. 35, Series E, No. 2, June 1968.

[7] J.R. Rice and G.F. Rosengren, Plane Strain Deformation Near a Crack Tip in a Power Law Hardening Material, J. Mech. Phys. Solids 16, 1968, p.1.

[8] J.W. Hutchinson, Singular Behavior at the End of a Tensile Crack in a Hardening Material, J. Mech. Phys. Solids 16 (1968) p. 13.

[9] J.W. Hutchinson, Plastic Stress and Strain Fields at a Crack Tip, J. Mech. Phys. Solids 16 (1968) p. 337.

[10] F.A. McClintock, Plasticity Aspects of Fracture, in: Chapter 2 of Fracture, An Advanced Treatise, Vol. III (H. Liebowitz, ed.), Academic Press N.Y., 1971.

[11] J.R. Wilshaw, C.A. Rau and A.S. Tetelman, A General Model to Predict the Elastic-Plastic Stress Distribution and Fracture Strength of Notched Bars in Plane Strain Bending, Engr. Fracture Mech. 1 (1969) p. 191.

[12] F.A. McClintock, Crack Growth in Fully Plastic Grooved Tensile Specimens, in: Physics of Strength and
Plasticity (A.S. Argon, ed.) MIT Press, 1969, p. 307.

[13] J.R. Rice and M.A. Johnson, The Role of Large Crack Tip Geometry Changes in Plane Strain Fracture, in: Inelastic Behavior of Solids (M.F. Kanninen et al., eds.), McGraw Hill, N.Y., 1970.

[14] P.V. Marcal and I.P. King, Elastic-Plastic Analysis of Two-Dimensional Stress Systems by the Finite Element Method, Int. J. Mech. 9 (1967) 3.

[15] J.L. Swedlow, A.H. Yang and M.L. Williams, ElastoPlastic Stresses and Strains in a Cracked Plate, in: Proc. Int'1. Conference on Fracture, Sendai, 1965, Vol. 1, pp. $259,1966$.

[16] J.L. Swedlow, Elasto-Plastic Cracked Plated in Plane Strain, Int. J. Fracture Mech. 5 (1969) pp. 33.

[17] N. Levy, P.V. Marcal, W.J. Ostegren and J.R. Rice, Small Scale Yielding Near a Crack in Plane Strain: A Finite Element Analysis, Int. J. Fracture Mech., in press.

[18] P.D. Hilton and J.W. Hutchinson, Plastic Intensity Factor for Cracked Plates, Engineering Fracture Mechanics, in press.

[19] D.J. Ayres, A Numerical Procedure for Calculating Stress and Deformation Near a Slit in a Three-dimensional Elastic-Plastic Solid, Eng. Fracture Mech., 2 (1970) p. 82.

[20] N. Levy and P.V. Marcal, Three-Dimensional ElasticPlastic Stress and Strain Analysis for Fracture Mechanics, Part I, Simple Flawed Specimens, HSSTP-TR-12, Brown University, Providence, Rhode Island

[21] O.C. Zienkiewicz and Y.K. Cheung, The Finite Element Method in Structural and Continuum Mechanics, McGrawHill Publishing Co., Ltd., 1967.

[22] D. Young, The Numerical Solution of Elliptic and Parabolic Partial Differential Equations, Survey of Numerical Analysis, J. Todd, ed., McGraw-Hill Publishing Co., Ltd., 1962.

[23] J.G. Sullivan, Fortran Input/Output for Tapes and Direct Access Devices, ORNL-TM-2142, February 1968.

[24] N. Levy, Application of the Finite Element Method to Large Scale Elastic-Plastic Problems of Fracture Mechanics, Ph.D. Thesis, Brown University, June 1970.

[25] G.R. Irwin, Fracture Mechanics, Structural Mechanics, (Proc. of First Naval Symp.), J.N. Goodier and N.J. Hoff, eds., Pergamon Press, 1960.

[26] B. Gross and J.E. Srawley, "Stress Intensity Factor for $r$ Single Edge Notch Specimens in Bending or Combined Bending and Tension by Boundary Collocation of a Stress Function," NASA Tech. Note D-2603, 1965. 